\title{
STRETCH: a Sensor Platform for Non-Invasive Remote Monitoring of Older People in Real Time
}

\author{
Mohamed Bennasar ${ }^{1}$, Ciaran McCormick ${ }^{1}$, Blaine Price ${ }^{1}$, Daniel Gooch ${ }^{1}$, Avelie \\ Stuart $^{2}$, Vikram Mehta ${ }^{1}$, Linda Clare ${ }^{2}$, Amel Bennaceur ${ }^{1}$, Jessica Cohen ${ }^{4}$, Arosha \\ Bandara $^{1}$, Mark Levine ${ }^{2}$ and Bashar Nuseibeh ${ }^{1,3}$ \\ ${ }^{1}$ School of Computing and Communications, The Open University, UK \\ ${ }^{2}$ Psychology, University of Exeter, UK \\ ${ }^{3}$ University of Limerick, Ireland \\ ${ }^{4}$ Age UK Exeter, UK
}

\begin{abstract}
The population of older adults is increasing across the globe; this growth is predicted to continue into the future. Most older adults prefer to live in their own home, but many live alone without immediate support. Living longer is often coupled with health and social problems and difficulty managing daily activities. Therefore, some level of care is required, but this is costly. Technological solutions may help to mitigate these problems by recognising subtle changes early and intervening before problems become unmanageable. Understanding a person's usual behaviour when carrying out Activities of Daily Living (ADL) makes it possible to detect and respond to anomalies. However, current commercial and research monitoring systems do not an analysis of ADL and are unable to detect subtle changes. To address this gap, we propose the STRETCH (Socio-Technical Resilience for Enhancing Targeted Community Healthcare) sensor platform that is comprised of non-invasive sensors and machine learning techniques to recognise changes and allow early interventions. The paper discusses design principles, modalities, system architecture, and sensor network architecture.
\end{abstract}

Keywords: Smart House, Sensor Platform, Older People care, Ambient Assisted living, eHealth.

\section{Introduction}

Advances in medical care, as well as growing awareness about the value of healthy nutrition and exercise, has led to a significant increase in life expectancy, particularly in developed countries. At the same time, the birth rate has fallen in most of these countries resulting in an aging population [1]. According to the UK Office of National Statistics, $18.2 \%$ of the population are aged 65 years and over and is expected to rise to $24 \%$ by 2037 ; by 2066 there will be 20.4 million people aged 65 years and over [2]. This growth in the aging population will be coupled with increases in health and social problems such as disability, chronic diseases and conditions, and social isolation and loneliness and will significantly increase pressure on health and social care services 
challenging their viability. Moreover, the cost of these services is increasing every year [3]. According to Age UK about 35\% of all people aged over 65 live [4]. Most of them require some degree of support in their daily life, which is usually provided by family members, and friends who form together what is called a circle of support [5]. Therefore, there is a growing need to develop cost effective tools and systems that can support and monitor older people at home without interrupting their daily routine, allowing them to stay at home and live independently while they are being monitored remotely $[1,6]$. Although the concept of smart houses has been around for several decades, smart home technology has gained a great deal of attention recently from researchers around the world [7].

Smart houses employ a variety of sensors depending on the focus and goals of the system and may include wearable sensors, Passive Infra-Red (PIR), environmental sensors, power consumption sensors, water consumption sensors, Radio-Frequency Identification (RFID) sensors, and cameras [8]. This paper introduces a new sensor platform for monitoring older adults at home as part of the STRETCH (Socio-Technical Resilience for Enhancing Targeted Community Healthcare) project, an EPSRC-funded multidisciplinary research project, led by The Open University in collaboration with University of Exeter and Age UK. The overall aim is to develop a smart system for enhancing care quality, safety, and security for older adults that can improve their ability to live independently in their own homes. The STRETCH system employs only noninvasive sensors, involves sophisticated data analyses, and improves the resilience of caregivers to enhance the resilience and adaptability of the circle of support. The ultimate target is to have the STRETCH sensor platform deployed in the houses of 25 participants; this paper discusses only the sensing layer of this system.

The main contribution of this paper lies in reporting the practical experience of designing and deploying the platform. To achieve this objective, design choices and technical details will be explained in detail with a special focus on architecture and system implementation.

The rest of this paper is structured as follows: section 2 discusses the related work; section 3 presents and explains the STRETCH sensor platform and the design choices inherent in the design; and section 4 concludes the paper with a discussion of the planned future work.

\section{Related work}

During the last decade, several smart home systems have been proposed for a variety of applications [9]. Most of the proposed systems follow the concept of ubiquitous sensing, where an integrated network of various sensors is used to collect data about the environment and the participants. These systems usually monitor the environment and the individuals to detect activities, falls, location, health status, and medicine intake [10]. The Department of Electronics at Tampere University of Technology proposed a sensor platform for human activity recognition. The platform relies on an indoor positioning system; the strength of the signal received by the receiver is used as a measure to predict the position of the participant [11]. The Great Northern Heaven Smart Home 
is a system that has been developed in Ireland [12]. The system employs sensors including: PIR sensors; contact sensors on windows and doors; light switches sensors; temperature sensors; light sensors; and sensors for detecting weather outside. A wired network is used to connect all the sensors to a local SQL server at the house. Aurama awareness is smart house system developed to support older people. It employs a tablet as a photo frame to communicate information with caregivers about the presence of the older adult [13]. It also employs an RFID sensor, and bed sensors to study the sleep and home occupancy. The SPHERE project (upon which some of the STRETCH sensing technology is based) uses multimodality sensing employing wearables, environment monitoring, and vision-based monitoring. This project aims to fuse sensor data to recognise and monitor different health problems. The sensor data is stored in a local computer at each house [14].

Pol et al. used a sensor monitoring system to study the perspectives and Activities of Daily Living (ADL) of older adults in their own homes. The system employs 16 simple binary sensors which include: toilet flushing sensor, PIR sensor, and magnetic contact sensor on doors and cabinets. They used machine learning techniques to analyse the data and detect simple activities. The collected sensor data is used to generate a report for health care professionals [15].

There are other systems that have been reported in the literature which focus on specific applications such as: fall detection [16]; medications intake [17]; assessing medical status 18]; assisting individuals with mild cognitive impairment [19], and assessing people living with Alzheimer's disease by monitoring them during the performance of daily tasks. The later system allows an assistant to send a prompt remotely when the individual makes any mistake during task performance [20].

Despite these various developments, in a systematic review of the use of smart home technology for detecting and managing ADL, Pol et al. (2013) argue that the evidence for validating and customising the application of sensing systems for communitydwelling (at home) older adults in their own homes is still scarce [21].

\section{STRETCH sensor platform design and implementation}

Several principles were considered in the STRETCH system design which have not previously been addressed together in single system of this kind in the literature, including:

1- It employs inexpensive hardware, open source software, and algorithms developed by the project team.

2- The platform employs only non-invasive modalities, and makes no use of any cameras or microphones.

3- The platform employs sensors which are very efficient in power consumption, and require the minimum amount of attention and interaction from the participant (long life batteries that do not require recharging and a system that is remote administered).

4- The system streams data in real-time to be able to provide information to caregivers when it is required. 
5- The minimum number of sensors are used to perform the required monitoring to reduce impact on the home.

6- The older adult participant has control over switching the platform on and off, and control over who has access to the data.

7- The developed system is easy to set up, requiring minimum technical expertise.

8- The system is adaptable to variations in layout of houses, type of material, and participant health and mobility conditions.

The developed sensor platform is designed to monitor a set of ADL which includes activities carried out in the person's living room, kitchen, bathroom, and bedroom. This set of ADLs will be used to analyse the older person's behaviour. The developed platform comprises various sensing modalities that can be categorised into three groups: body worn low-power sensors; environmental sensors; and power consumption sensors. Figure 1 shows a high level view of the system architecture. The STRETCH platform is made up of three layers: a sensing network; central data analysis; and intervention layer. The data collected from the sensors is transmitted to the central server via internet by the gateway.

\subsection{Sensors}

This section briefly discusses the sensing modalities employed in the STRETCH platform. The platform uses seven types of sensors. Five are for monitoring the environment: temperature sensor, humidity sensor, brightness sensor, barometric pressure sensor, and PIR sensor; these are combined in one device called an environmental sensor. The wearable sensor is used to monitor participants' movement, and the electricity sensor is used monitor the power consumption of the entire house. More details about the hardware components used within the developed platform are descried in the following sections:

\section{Environmental Sensor:}

This device has been developed as a part of the Sensor Platform for Healthcare in Residential Environment (SPHERE) project [22]. Figure 2 (b) shows the device. It was chosen because of its power efficiency and wireless connectivity. It is a cluster of sensors, which consist of temperature, humidity, lamination, air pressure and PIR sensors. It provides information about the surrounding environment. The time- stamped sensor readings are periodically transmitted wirelessly through the mesh network to a relay device. All readings have a pre-defined message format. The physical dimensions are $85 \times 85 \times 25 \mathrm{~mm}$, and it is powered by a $3.6 \mathrm{~V}, 2600 \mathrm{mAh}$ Lithium Thionyl Chloride AA battery which lasts 6 months. Every room in the house is fitted at least with one sensor. 


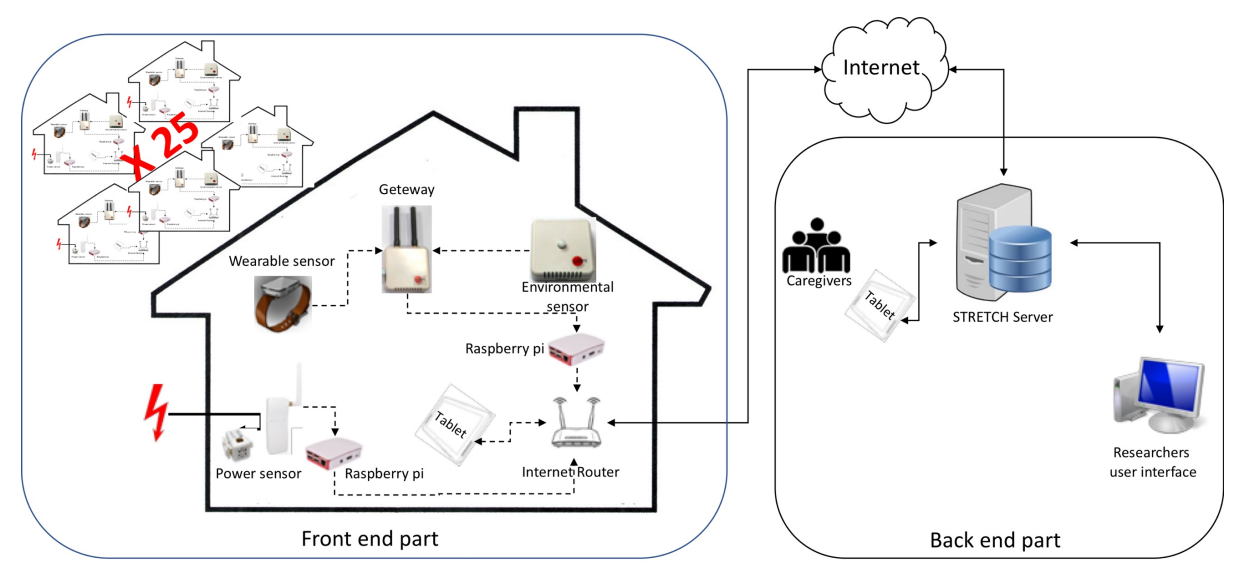

Fig. 1. STRETCH system architecture

\section{Wearable Sensor:}

A SPHERE wrist-worn wearable sensor [23] is used for on-body sensing in the STRETCH sensor platform. This device is selected because of its power efficiency and its wireless connectivity in real-time. The wearable consists of two tri-axial accelerometers to measure participant hand acceleration. The sensor is powered by $100 \mathrm{mAh} \mathrm{Li-}$ Po rechargeable battery (which lasts about 20 days). The physical dimensions of the sensor are $41 \times 22 \times 8 \mathrm{~mm}$. It connects to the architecture using Bluetooth Low Energy which is less than half the energy consumption of ZigBee. The device periodically broadcasts the sensor readings in a time stamped format of the acceleration in three axes with sampling frequency of $25 \mathrm{~Hz}$.

\section{Power Consumption Sensor:}

For whole home power consumption monitoring we use an off the shelf consumer consumption monitoring system, the OWL Intuition-e. This system consists of two parts, a transmitter unit (OWL transmitter) and an internet connected gateway (OWL gateway).

A standard current clamp sensor is placed around the wire that feeds electricity into the home. In the UK, this wire is found coming out of the premises' electricity meter. The current sensor is connected to the transmitter unit using a $3.5 \mathrm{~mm}$ jack. The transmitter sends the current energy consumption at 12 second intervals to the internet connected gateway unit over $433 \mathrm{MHz}$. The transmitter is powered using 3 standard AA alkaline batteries which last over 14 months.

The OWL gateway is powered by mains power and connects to the internet via ethernet. In its default configuration it sends the energy readings to OWL's cloud platform which allows consumers to view their energy usage data but our software listens for the multicast transmissions on the local network and sends the data to our server. 


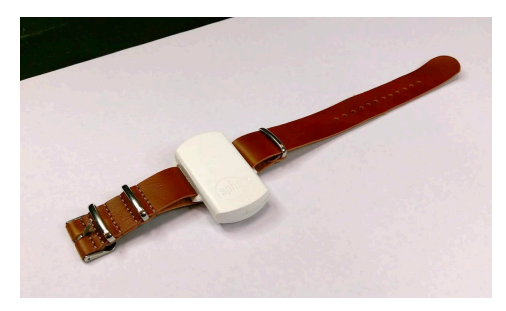

a

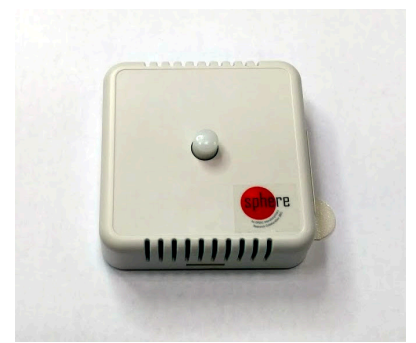

b

Fig. 2. (a) SPHERE wearable sensor, (b) SPHERE Environmental sensor

\subsection{Sensor Network Architecture}

In this section, we describe how we were able to collect the data that was generated by the local sensors and send it to the centralised STRETCH Management System (SMS) and discusses the challenges that had to be overcome carry this out.

In the previous section we indicated that data is collected using the SPHERE sensors and an off-the-shelf power consumption sensor. The data is gathered locally using a Raspberry Pi 3B+ running a Linux variant. The sensors send their data via a series of relay devices to a root gateway that is connected to the Raspberry Pi. These data were broadcast locally using the light weight publish-subscribe messaging protocol, Message Queuing Telemetry Transport (MQTT). Unlike the SPHERE sensor platform where the data is stored locally, in the STRETCH platform the data is transmitted in almost realtime to SMS. This has been achieved by setting up a remote Eclipse Mosquitto MQTT broker that could communicate with the local broker. The broker is password protected to prevent unwanted messages. The local SPHERE Raspberry Pi has been reconfigured to form a bridge with the remote broker, all sensor messages are sent to the local broker and then send over the internet to the remote SMS.

The message handling component of SMS is written in python using the Django Web Framework (Django). Django was used as it provides a powerful Object-Relational Mapping (ORM) layer that simplifies the reading and writing of data to the underlying PostgreSQL database.

Participants and their caregivers will access the data and aggregated information via a custom smartphone app while the research team uses a web user interface using a RESTful API. The Django REST Framework (DRF) was used to provide a Restful API that is allows us to build user-interfaces for the researchers and the participants. These were chosen as they provided a developer-friendly way of interacting with databases and building robust RESTful APIs.

SMS runs on a Virtual Private Server with 4 vCPUs, $8 \mathrm{~GB}$ of memory and $60 \mathrm{~GB}$ of disk space, running Ubuntu 16.04.3.

On average, e a house containing 10 environmental sensors, one wearable and one power consumption sensor will produce around 55,000 environmental readings, 13,400 wearable readings and 300 energy consumption readings per hour. Due to the amount of data produced a clean-up task is run once a day ensuring only 10 days' worth of data is kept in the database. 


\subsection{System Data Loss}

A number of factors can cause data loss, including faulty sensors which may stop sending data, or send corrupt readings. Therefore sensor data validation is needed which will be discussed in the next section.

Verbose logging can be another factor in data loss. When one of the scripts that runs periodically fails, this can add a massive amount of data to the log files, which leads to rapid increase in the used memory on the server, and consequently to a crash.

Data loss can occur at different points within the system. It usually occurs at points where data are either transferred or processed. At the local level, data loss can happen at the sensor (the sensor malfunctions), during the relaying of the data to the Raspberry $\mathrm{Pi}$ (communication errors between the sensor and the relay/root gateways) or when the data is published by the MQTT broker.

Due to a software issue in the SPHERE environmental sensors, 30 PIR readings are sent every 20 seconds with the last 10 of one message and the first 10 of the next message being identical. When processing a PIR message we bulk inserted all the PIR readings into the database. This meant that $50 \%$ more data $(90$ readings/minute vs. 60 readings/minute) was being inserted into the database than required. With a small deployment of 10 houses with 5 environmental sensors this would result in 2.16 million more readings in the database per day than required.

Due to this problem, the PIR readings are checked to see if they already exist in the database for a particular device id and timestamp and if not it is inserted. This means that for each message we had to iterate over the 30 readings, individually performing the update_or_create, which was very slow and lead to data loss. The underlying PostgreSQL database, however supported raw SQL "upserts" (update or insert). To overcome the data loss we re-implemented the PIR message handling using raw SQL upsert statements.

With regards to the power consumption sensor we expect data to be sent at 12 second intervals but have noticed that in low/uniform consumption circumstances data can be sent at 60 second intervals. The system is able to handle both of these situations.

Data can also be lost during data processing. If the system detects malformed data, the data are thrown away and an error message is logged. These messages are periodically viewed by researchers to improve the robustness of the system. Due to message publication patterns there are large periods where no messages are received and then short periods where large volume of messages are received. This is handled by placing the messages in a queue that can be handled by multiple worker processes in a parallel manner.

\subsection{Sensor Data Validation}

Every sensor sends a heartbeat message which contains device information such as battery voltage level. SMS contains a device monitoring component that periodically (every 5 minutes) checks the last time a device heartbeat message was received. If the system detects that a device has not "phoned home" in the last 5 minutes a message is sent to several members of the research team to investigate. 
Raw sensor data validation is an important step in minimising data loss, making sure that every sensor is working properly, and ensuring that transmitted data is correct. One basic check is to recognize any reading outside the logical expected range.

More checks can be performed in a variety of ways depending on the transmitted pattern of sensor data. Data validation for periodic sensors is the easiest, such as wearable, power, and PIR sensors. The wearable sensor sends 25 time-stamped readings per second; if there are significantly fewer this may indicate a problem with the sensor or with the wireless network. The same situation holds for the PIR sensor and power sensor which send a reading every second and every 12 seconds respectively.

\subsection{Hardware Monitoring and Sensor Data Access}

A prototype Graphical User Interface (GUI) has been developed (shown in figure 3). It displays live sensor data for every house. It is designed to give the data analysts the ability to export the raw data from any of the sensors. Moreover, the GUI displays the status of each sensor in the house.

The GUI was developed using the Angular 7.1, a front-end web application framework based on the TypeScript language. It retrieves data from SMS using REST API calls.

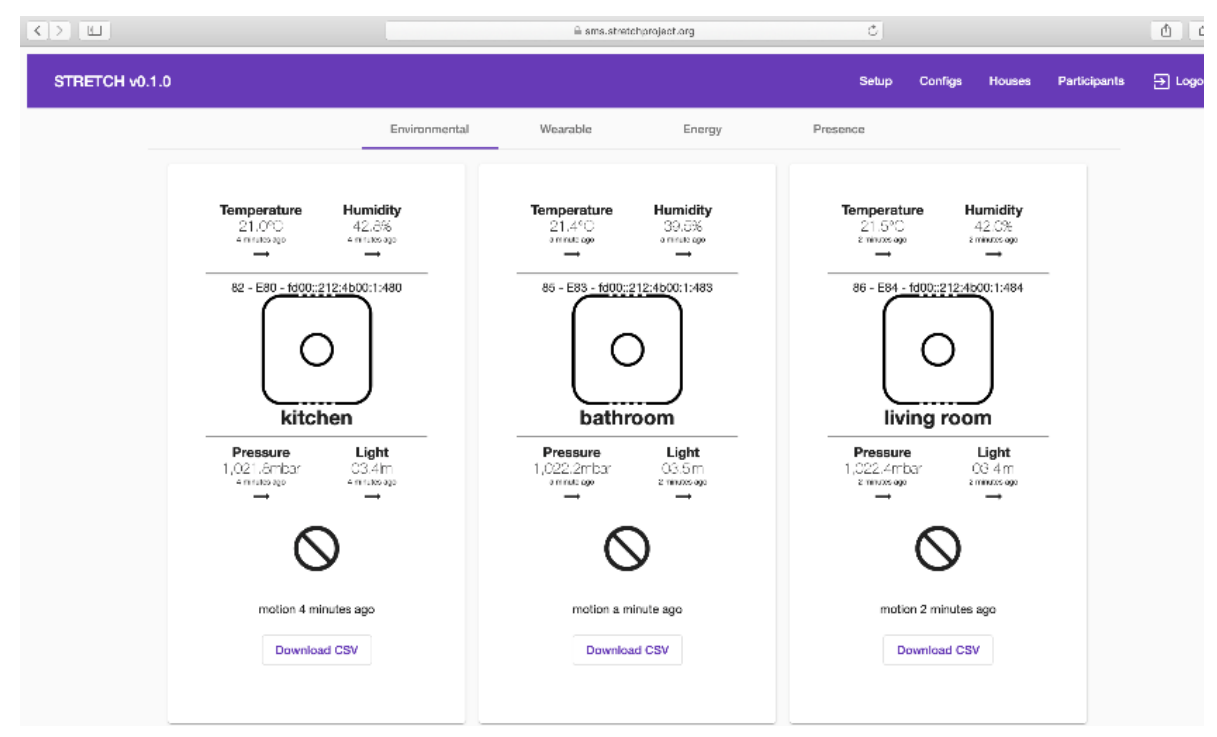

Fig. 3. Prototype GUI

\section{Conclusions and future work}

Most of the current commercial systems are designed to send sensor measurements to a central monitoring centre, where a human operator intervenes when a sensor reading is above a specific threshold. Many automatic sensor platforms have been proposed to support the care of older people. Most of these are for monitoring specific activities 
such as fall detection, medication intake, and detecting emergency situations, and they do not involve carers. The STRETCH platform uniquely facilitates the integration of data from previously developed sensors, transmits them in real-time, and enables the sharing of information about ADLs, which could be developed into a wider variety of applications than in previous systems. This paper describes the decisions made during the design and implementation stages which can be used as a guide by other researchers for developing remote sensing and security systems.

Our future research plan includes studying wider deployment issues, annotated data collection, developing participant app, and developing AI techniques for activity recognition, behaviour analysis and anomaly detection. Furthermore, it will also include studying system adaptation and both technical and social resilience.

\section{Acknowledgements}

This research was part funded by UK EPSRC grants EP/P01013X/1 (STRETCH) and EP/R013144/1 (SAUSE) and ERC grant 291652 (ASAP)

\section{References}

1. Majumder, S., Aghayi, E., Noferesti, M., Memarzadeh-Tehran, H., Mondal, T., Pang, Z. and Deen, M., Smart homes for elderly healthcare-Recent advances and research challenges. Sensors, 17(11), p.2496 (2017).

2. Office for National Statistics. Overview of the UK population: November 2018. from https://www.ons.gov.uk/releases/ overviewoftheukpopulationnovember2018. (November 2018).

3. Fiorini, L., Cavallo, F., Dario, P., Eavis, A. and Caleb-Solly, P., Unsupervised machine learning for developing personalised behaviour models using activity data. Sensors, 17(5), p.1034 (2017).

4. Age UK. Later Life in the United Kingdom. From https://www.ageuk.org.uk/globalassets/age-uk/documents/reports-and-publications/later_life_uk_factsheet.pdf. (April 2018).

5. Anderson, G. and Knickman, J.R. Changing the chronic care system to meet people's needs. Health Affairs, 20(6), pp.146-160 (2001).

6. Aramendi, A.A., Weakley, A., Goenaga, A.A., Schmitter-Edgecombe, M. and Cook, D.J. Automatic assessment of functional health decline in older adults based on smart home data. Journal of biomedical informatics, 81, pp.119-130 (2018).

7. Sanchez, V., Pfeiffer, C. and Skeie, N.O. A review of smart house analysis methods for assisting older people living alone. Journal of Sensor and Actuator Networks, 6(3), p.11 (2017)

8. Dasios, A., Gavalas, D., Pantziou, G. and Konstantopoulos, C. July. Wireless sensor network deployment for remote elderly care monitoring. In Proceedings of the 8th ACM International Conference on PErvasive Technologies Related to Assistive Environments (p. 61). ACM, (2015).

9. Rashidi, P. and Mihailidis, A., 2013. A survey on ambient-assisted living tools for older adults. IEEE journal of biomedical and health informatics, 17(3), pp.579-590 (2013).

10. Alemdar, H. and Ersoy, C. Wireless sensor networks for healthcare: A survey. Computer networks, 54(15), pp.2688-2710 (2010). 
11. Valtonen, M., Vuorela, T., Kaila, L. and Vanhala, J. Capacitive indoor positioning and contact sensing for activity recognition in smart homes. Journal of Ambient Intelligence and Smart Environments, 4(4), pp.305-334 (2012).

12. Doyle, J., Kealy, A., Loane, J., Walsh, L., O'Mullane, B., Flynn, C., Macfarlane, A., Bortz, B., Knapp, R.B. and Bond, R. An integrated home-based self-management system to support the wellbeing of older adults. Journal of ambient intelligence and smart environments, 6(4), pp.359-383 (2014).

13. Dadlani, P., Markopoulos, P., Sinitsyn, A. and Aarts, E. Supporting peace of mind and independent living with the Aurama awareness system. Journal of Ambient Intelligence and Smart Environments, 3(1), pp.37-50 (2011).

14. Zhu, N., Diethe, T., Camplani, M., Tao, L., Burrows, A., Twomey, N., Kaleshi, D., Mirmehdi, M., Flach, P. and Craddock, I. Bridging e-health and the internet of things: The sphere project. IEEE Intelligent Systems, 30(4), pp.39-46 (2015).

15. Pol, M., van Nes, F., van Hartingsveldt, M., Buurman, B., de Rooij, S. and Kröse, B. Older people's perspectives regarding the use of sensor monitoring in their home. The Gerontologist, 56(3), pp.485-493.(2014).

16. Wang, C.C., Chiang, C.Y., Lin, P.Y., Chou, Y.C., Kuo, I.T., Huang, C.N. and Chan, C.T. Development of a fall detecting system for the elderly residents. In Bioinformatics and Biomedical Engineering. ICBBE 2008. The 2nd International Conference on (pp. 1359-1362). IEEE (2008).

17. Lopez-Nores, M., Pazos-Arias, J.J., Garcia-Duque, J. and Blanco-Fernandez, Y. January. Monitoring medicine intake in the networked home: The iCabiNET solution. In Pervasive Computing Technologies for Healthcare, 2008. PervasiveHealth 2008. Second International Conference on(pp. 116-117). IEEE (2008)

18. Pang, Z., Chen, Q. and Zheng, L., 2009, November. A pervasive and preventive healthcare solution for medication noncompliance and daily monitoring. In Applied Sciences in Biomedical and Communication Technologies. ISABEL 2009. 2nd International Symposium on (pp. 1-6). IEEE (2009).

19. Seelye, A.M., Schmitter-Edgecombe, M., Cook, D.J. and Crandall, A. Naturalistic assessment of everyday activities and prompting technologies in mild cognitive impairment. Journal of the International Neuropsychological Society, 19(4), pp.442-452 (2013).

20. Lapointe, J., Bouchard, B., Bouchard, J., Potvin, A. and Bouzouane, A. June. Smart homes for people with Alzheimer's disease: adapting prompting strategies to the patient's cognitive profile. In Proceedings of the 5th international conference on pervasive technologies related to assistive environments (p. 30). ACM (2012).

21. Pol, M.C., Poerbodipoero, S., Robben, S., Daams, J., van Hartingsveldt, M., de Vos, R., de Rooij, S.E., Kröse, B. and Buurman, B.M. Sensor monitoring to measure and support daily functioning for independently living older people: a systematic review and road map for further development. Journal of the American Geriatrics Society, 61(12), pp.2219-2227. (2013).

22. Woznowski, P., Fafoutis, X., Song, T., Hannuna, S., Camplani, M., Tao, L., Paiement, A., Mellios, E., Haghighi, M., Zhu, N. and Hilton, G, June. A multi-modal sensor infrastructure for healthcare in a residential environment. In Communication Workshop (ICCW), 2015 IEEE International Conference on(pp. 271-277). IEEE (2015). 